\title{
Optimized phase field model for diblock copolymer melts
}

\author{
Jimmy V. Liu, ${ }^{\dagger, \ddagger}$ Carlos J. García-Cervera, ${ }^{\Phi, \S}$ Kris T. Delaney, ${ }^{\ddagger}$ and Glenn H. Fredrickson*, ${ }^{*}, \ddagger, \|$ \\ $\dagger$ Department of Chemical Engineering, University of California, Santa Barbara, Santa Barbara, California 93106, United \\ States \\ $\ddagger$ Materials Research Laboratory, University of California, Santa Barbara, Santa Barbara, California 93106, United States \\ IDepartment of Mathematics, University of California, Santa Barbara, Santa Barbara, California 93106, United States \\ $\S$ Visiting Professor at BCAM-Basque Center for Applied Materials, Mazarredo 14, E48009 Bilbao, Basque Country, Spain \\ ||Materials Department, University of California, Santa Barbara, Santa Barbara, California 93106, United States \\ E-mail: ghf@ucsb.edu
}




\section{Supporting Information}

The cost function $\Psi$ in eq 18 requires evaluating the force and stress for SCFT and the phase field model. For SCFT, the force and stress are both zero by design when evaluated at $\phi=\phi^{*}$. For the phase field model described in eq 13 , the force can be computed by taking the functional derivative as follows:

$$
\frac{1}{C} \frac{\delta H_{\mathrm{r}}[\phi]}{\delta \phi(\mathbf{r})}=2 c_{2} \delta \phi(\mathbf{r})+3 c_{3} \delta \phi(\mathbf{r})^{2}+4 c_{4} \delta \phi(\mathbf{r})^{3}-2 c_{5} \nabla^{2} \phi(\mathbf{r})+2 c_{6} \int d \mathbf{r}^{\prime} G\left(\mathbf{r}-\mathbf{r}^{\prime}\right) \delta \phi\left(\mathbf{r}^{\prime}\right),
$$

where we have omitted boundary terms that vanish under periodic boundary conditions. To compute the stress, we make explicit a scale factor $L$ for the system size, then take the derivative of $H_{\mathrm{r}, V}$ with respect to the isotropic strain $\epsilon=\frac{L-L_{0}}{L_{0}}$. The resulting expression is

$$
\frac{1}{C} \frac{\partial H_{\mathrm{r}, V}[\phi]}{\partial \epsilon}=\frac{1}{(\epsilon+1) V} \int d \mathbf{r}\left\{-2 c_{5}|\nabla \phi(\mathbf{r})|^{2}+2 c_{6} \int d \mathbf{r}^{\prime} G\left(\mathbf{r}-\mathbf{r}^{\prime}\right) \delta \phi(\mathbf{r}) \delta \phi\left(\mathbf{r}^{\prime}\right)\right\}
$$

Figure S1 shows the residuals of OPF model parameters from the regression to phase field mapping data. When scaled by the magnitude of the relevant parameter, we found that the errors were everywhere within $2 \%$ for $\chi N-\chi N_{\mathrm{s}}>3$.

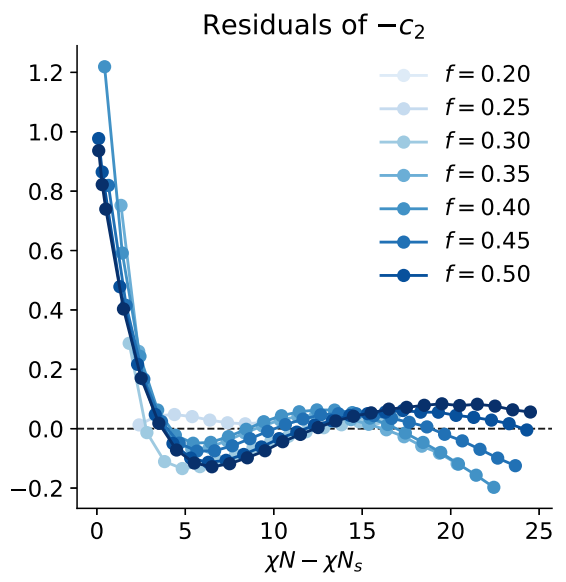

Residuals of $c_{5}$

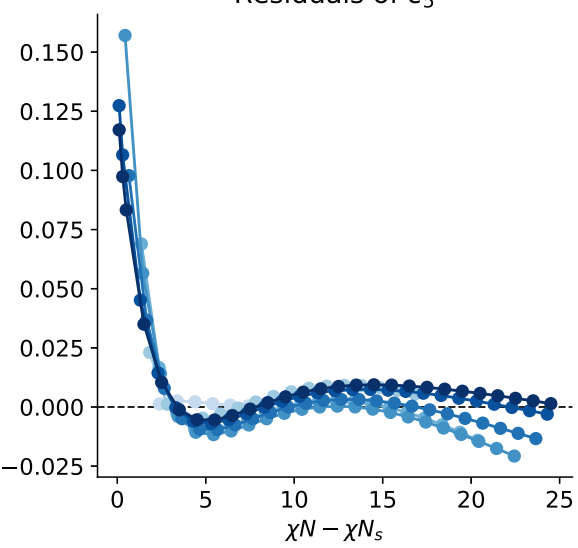

Residuals of $-c_{3}$

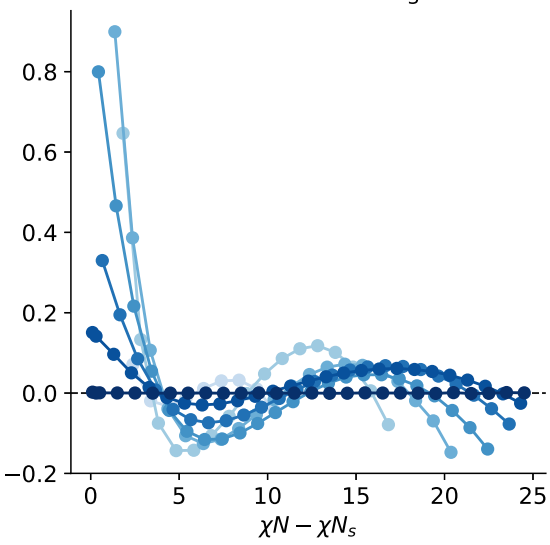

Residuals of $c_{6}$

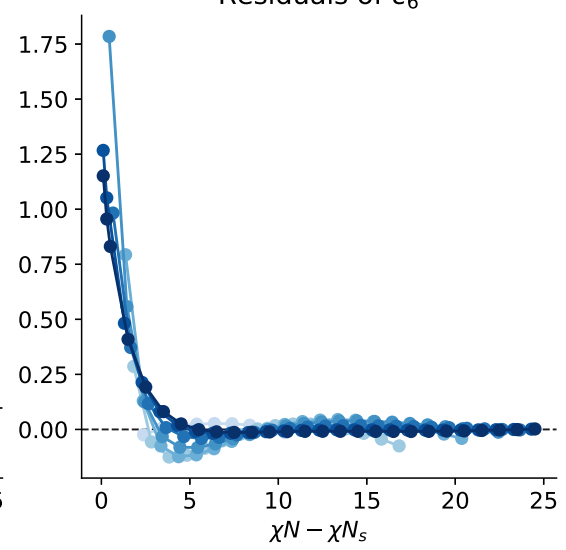

Residuals of $c_{4}$

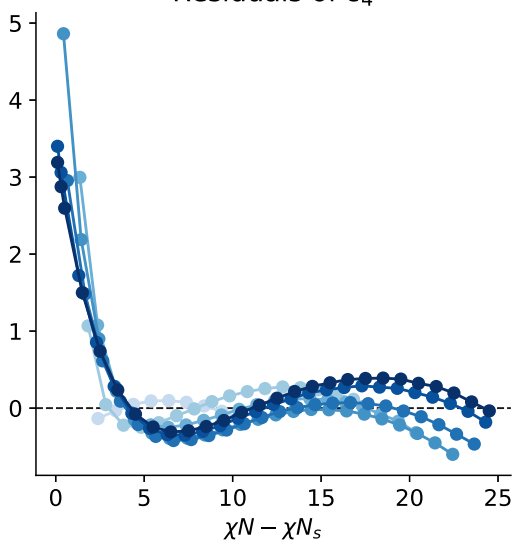

Residuals of $c_{6} / c_{5}$

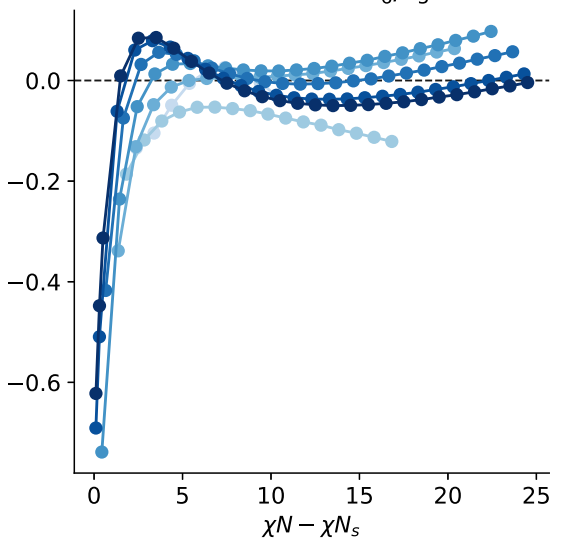

Figure S1: From left to right: residuals of OPF model parameters $-c_{2},-c_{3}, c_{4}, c_{5}, c_{6}$, and the ratio $c_{6} / c_{5}$ as functions of $\chi N$ for block fractions $f$ from 0.5 (darkest) to 0.2 (lightest) from regression to data obtained by phase field mapping. 
Figure S2 shows coefficients for OPF-HEX, the model obtained by phase field mapping from the HEX phase. Trends in both $\chi N$ and $f$ match those for the mapping performed in the lamellar phase.
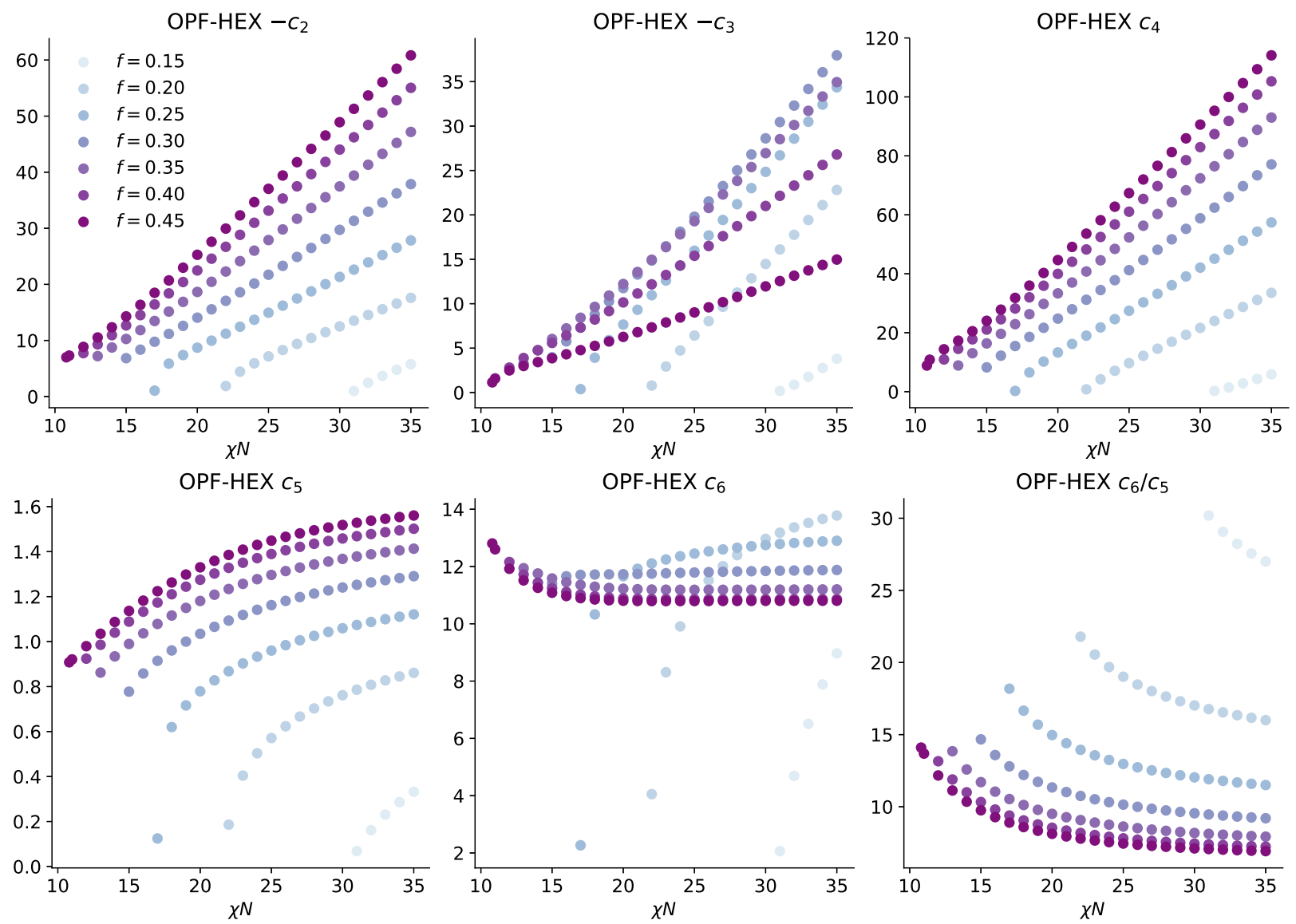

Figure S2: From left to right: OPF-HEX model parameters $-c_{2},-c_{3}, c_{4}, c_{5}, c_{6}$, and the ratio $c_{6} / c_{5}$ as functions of $\chi N$ for block fractions $f$ from 0.5 (darkest) to 0.2 (lightest) obtained from phase field mapping (points) and values from the Ohta-Kawasaki model (dashed lines). 
Figure S3 shows representative stress-free equilibrium density fields for SCFT and for the OPF and OK models for different values of $f$ and $\chi N$. We observe that the OPF model is a better approximation of SCFT than the OK model, both in terms of the commensurate domain spacing $L_{0, \mathrm{PF}}$ and the shape of the density profile. However, as the block asymmetry increases, both phase field models decrease in quality due to a weakness in the basis set of eq 13 .
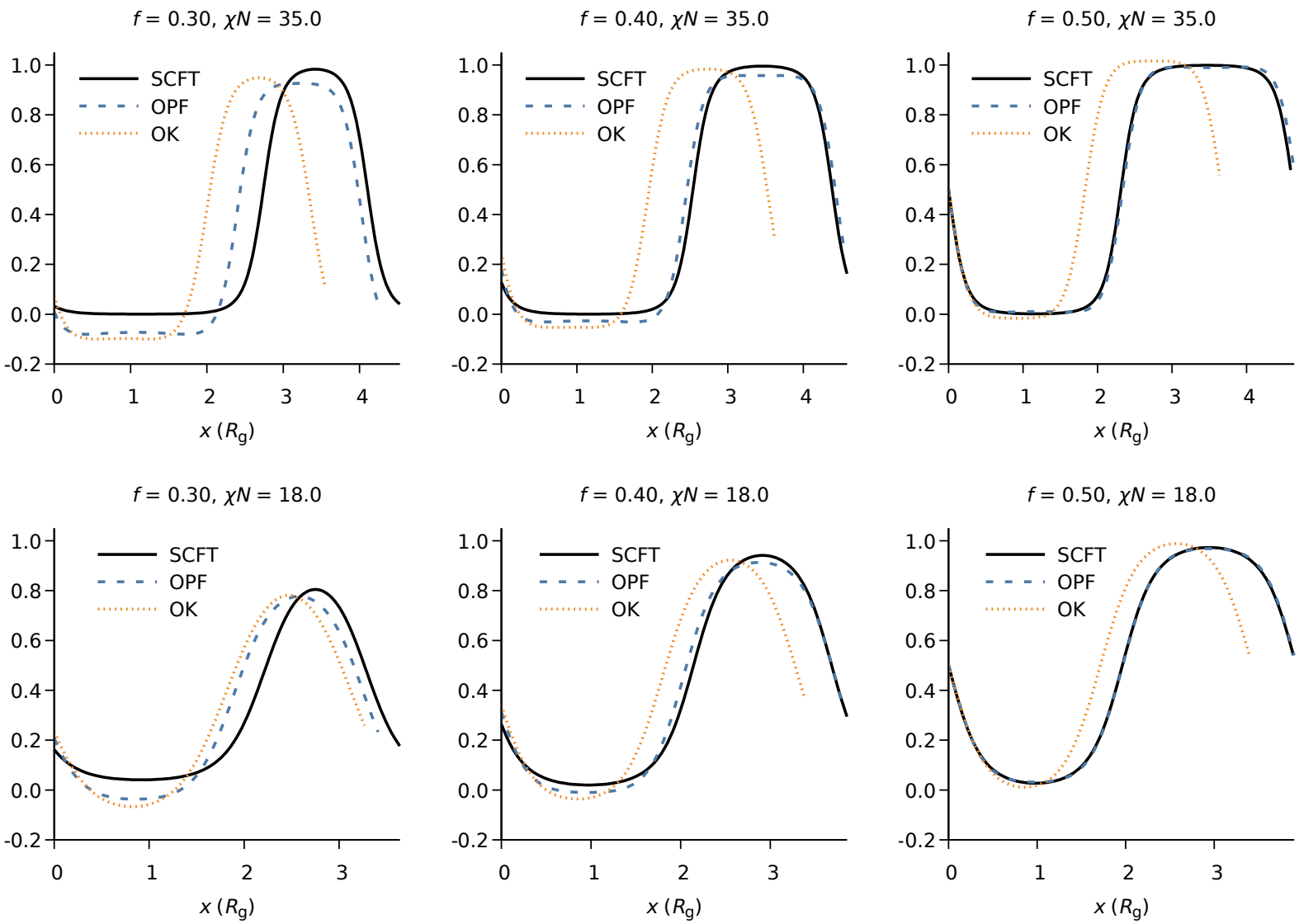

Figure S3: Representative stress-free equilibrium density fields for SCFT (solid), the OPF model (dashed blue), and the OhtaKawasaki model (dotted orange) for the lamellar phase for $f=0.3$ (left), $f=0.4$ (center), and $f=0.5$ (right), and at $\chi N=35$ (top) and $\chi N=18$ (bottom). 